\title{
Resource reduction via repeaters in entanglement distribution
}

\author{
A. Hutton and S. Bose \\ Centre for Quantum Computation, Clarendon Laboratory, University of Oxford, Parks Road, Oxford OX1 3PU, England
}

\begin{abstract}
We show that the amount of entanglement needed as an initial resource to set up a certain final amount of entanglement between two ends of a noisy channel can be reduced in certain cases by using quantum repeaters. Our investigation (for various channels) considers cases when a large number of entangled pairs are transmitted through the channel using known asymptotic results and conjectured bounds on distillable entanglement.
\end{abstract}

\section{INTRODUCTION}

The resource of entanglement [1] 3] has many useful applications in quantum information processing, such as secret key distribution [4], teleportation [5] and dense coding [6]. Recently, there has been an intense interest in the physical implementation of these quantum communication protocols [7]. In the future, it is conceivable that a large number of distant users would want to engage in communicating with each other through quantum protocols for purposes of security 4,8 , doubled capacity [6], high fidelity state transfer (by teleportation [5]), reduced communication complexity [9], secret sharing [10], linking distant quantum computers |11], for distributed computation [12,13 and a host of other applications 114,15. For this to happen, any pair or group of distant users will need to share particles in any desired pure entangled state, irrespective of noise in the entanglement distribution channels. In this context, various general schemes which could directly or indirectly aid in the distribution of entanglement have been theoretically studied 12, 16 24 and experimentally demonstrated [25]. One of these schemes, suggested by Briegel et al. and Dür et al., is the idea of quantum repeaters [24]. In their scheme, a number of nodes called repeaters are placed between the ends of a noisy channel. The entanglement is first distributed between neighboring nodes and concentrated by local actions [16 19 at these nodes to produce a few highly entangled pairs. Then entanglement swapping 21 23. is used to connect the highly entangled pairs in series to obtain some highly entangled pairs between the ends of the noisy channel. In this way, the exponential degradation of entanglement with channel length can be prevented at the expense of only a logarithmic increase in resources (cost) and a polynomial increase in time [24]. In this paper, we will look at the use of repeaters from a rather different angle. We will show that if the amount of entanglement finally required between two ends of a noisy channel was fixed, it could be achieved, in certain circumstances, at a much lower cost using quantum repeaters.

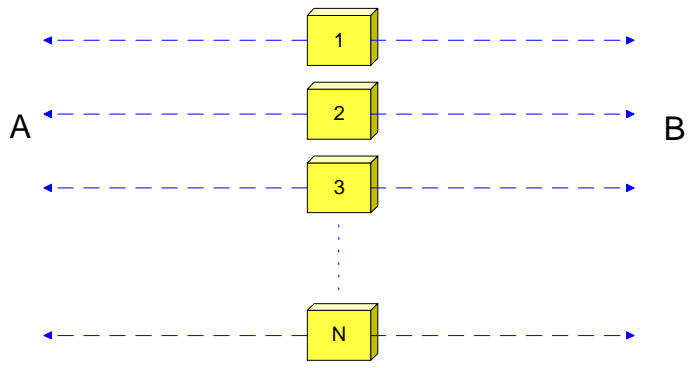

FIG. 1. The figure shows a specific way of distributing entanglement between two ends of a noisy channel. $N$ sources are placed at the midpoint of the channel and made to emit maximally entangled qubit pairs.

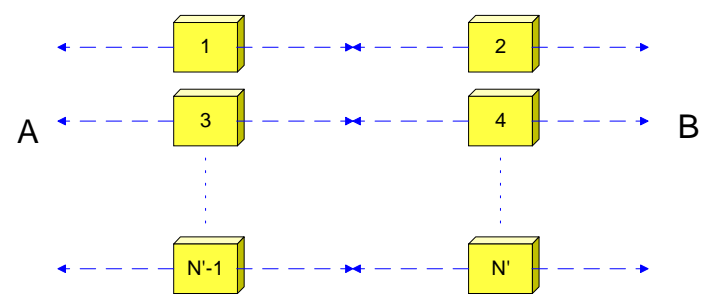

FIG. 2. The figure shows a way of distributing entanglement between two ends of a noisy channel which uses a quantum repeater positioned at the midpoint of the channel. With this configuration, $N^{\prime}<N$ sources of entangled pairs may be sufficient to establish as much entanglement between the ends of the channel as one without repeaters and $N$ sources.

Consider the following scenario. You are given $N$ boxes labeled $1,2, \ldots, N$, each of which can be made to emit exactly one maximally entangled particle pair on demand. Your target is to distribute entanglement between two 
ends $A$ and $B$ of a noisy channel. You have complete freedom of placing the boxes anywhere along the length of the channel prior to distribution. Suppose you first place all the $N$ sources at the midpoint of the channel as shown in Fig. 1 and then make them emit maximally entangled pairs. The pairs will lose part of their initial entanglement while traveling from the middle to the end of the channel. At the end of this process, a certain amount of entanglement is obtained between the ends $A$ and $B$ of the channel. You are then asked whether it is possible to devise a more effective scheme in which you distribute the same amount of entanglement between $A$ and $B$ using a lower number of initial sources of maximally entangled pairs. It turns out, as we will show, that the scheme shown in Fig.2 can accomplish this in certain circumstances (i.e. for certain lengths and types of the noisy channels). The basic idea is to divide the channel into two equal segments and place a repeater station at the midpoint of the channel. A lower number $N^{\prime}$ of sources may now be sufficient to distribute as much entanglement between $A$ and $B$ as before. We first have to place $N^{\prime} / 2$ sources at the midpoint of each half-channel. This is shown in Fig. 2 with boxes $1,3, \ldots, N^{\prime}-1$ having been placed at the midpoint of the left half-channel and boxes $2,4, \ldots, N^{\prime}$ placed at the midpoint of the other halfchannel, where $N^{\prime}<N$. Then they are made to emit maximally entangled pairs which are stopped at $A, B$ and the repeater station. After traversing a certain distance, each of the pairs would have lost part of their entanglement. An operator at the repeater station has to now act together with operators at $A$ and $B$ to locally distill two sets of maximally entangled pairs [17]: one set between the repeater and $A$, and the other set between the repeater and $B$. The operator at the repeater then connects these maximally entangled pairs by entanglement swapping 212 23] to obtain a set of maximally entangled states between $A$ and $B$. Though the maximum number of possible (entangled or disentangled) particle pairs between $A$ and $B$ is $N^{\prime} / 2<N$ in the case of distribution with a repeater, the degradation of entanglement of each pair is also less (as each particle now travels half the distance as before). The scheme with a repeaters saves resources when the positive effect of lower entanglement loss on traversal of the channel overrides the negative effect of having a lower number of entanglement sources to start with.

The resource reduction process described above, as we shall demonstrate, can be made more significant for certain channel lengths by dividing the channel into an even larger number of segments and placing repeater stations at the junctions of these segments. This would lead to an important cost reduction in the distribution of entanglement. The cost of distributing entanglement will become a very important issue in the future if quantum communications take off and this topic has already received serious attention (see, for example, Cirac et al. [12]). In this paper, we consider the cost reduction to be approxi- mately proportional to reduction of the initial resource of entanglement (in terms of the number of sources of maximally entangled pairs used). We neglect, for example, the cost of classical communication during the entanglement distillation procedures (though this cost might not necessarily increase on using repeaters, as the number of pairs distilled in parallel is also decreased). This assumption seems reasonable, as entanglement is, generally, the most expensive of all relevant resources, while classical communications can be accomplished through a conventional telephone line.

Our investigation differs in two distinct ways from the investigations in the original proposal for quantum repeaters [24]. Firstly, the focus is shifted from reliable distribution of entanglement to resource reduction. While in 24] it was shown that one could use repeaters to prevent the exponential degradation of entanglement with only logarithmic increase in cost, we show that the cost of distributing a certain fixed amount of entanglement between two ends of a channel can be reduced using repeaters. Secondly, we will take a general approach based on known values and bounds on distillable entanglement [17,26 29] and conjectured bounds [30]. In this sense, our analysis will remain valid even if improved (or even optimal) entanglement distillation schemes are found and even if the future advance of technology results in completely error free quantum operations. We should mention here that for those particular cases for which we use known conjectures, rather than proven results, our results may not continue to hold true if the conjecture is shown invalid. However, exactly the same methodology as ours can still be used to investigate resource reduction via repeaters using any accepted nonzero lower bounds on distillable entanglement. We should also mention that we consider only asymptotic distillation procedures, primarily because of they give simple entropic expressions for entanglement. This, of course, provides a restriction to the amount by which resources can be reduced while still keeping our analysis valid. We would require asymptotic distillation results to remain valid even for the sets of entangled pairs being distilled between two adjacent repeaters or a repeater and an end of the channel. This lower number of entangled pairs must thus, already, be very large. So we would want the resource reduction to be not so much as to make some of the distillation procedures nonasymptotic. For this reason, we will calculate resource reduction as a ratio instead of as a difference and it will remain valid as long as the initial number $N$ of sources is so large, that even during the reduced resource operation with repeaters, all the asymptotic results remain applicable.

In the next section we proceed to investigate resource reduction by repeaters for those channels which result in states whose distillable entanglement is exactly known. 


\section{EXACT RESULTS}

\section{A. Watched amplitude damping channel}

We will start with the watched amplitude damping channel, as the output states of this channel are pure and we can readily use the known asymptotic distillation results for pure states [16]. The amplitude damping channel has the following effect on states $|0\rangle$ and $|1\rangle$ of a qubit [2, 3]:

$$
\begin{aligned}
|0\rangle|0\rangle_{E} & \rightarrow|0\rangle|0\rangle_{E} \\
|1\rangle|0\rangle_{E} & \rightarrow \sqrt{1-p}|1\rangle|0\rangle_{E}+\sqrt{p}|0\rangle|1\rangle_{E}
\end{aligned}
$$

where $p$ is a parameter that is related to the length of the channel by

$$
p=1-e^{-2 \Gamma}
$$

in which $\Gamma$ is a quantity proportional to the length of the channel and the subscript $E$ stands for the environmental state. The expression for $p$ above has been so chosen that at infinite length of the channel, the state of the system becomes $|0\rangle$. When the environment is being watched, and found to be in the state $|0\rangle_{E}$, we can consider the evolution of the state to be given by [3]

$$
\begin{aligned}
& |0\rangle \rightarrow|0\rangle \\
& |1\rangle \rightarrow e^{-\Gamma}|1\rangle
\end{aligned}
$$

and this occurs for a state $\alpha|0\rangle+\beta|1\rangle$ with the probability $\frac{|\alpha|^{2}+|\beta|^{2} e^{-2 \Gamma}}{|\alpha|^{2}+|\beta|^{2}}$. We consider sending qubits in two types of initial states down the channel, namely

$$
\frac{1}{\sqrt{2}}(|01\rangle+|10\rangle) \text { and } \frac{1}{\sqrt{2}}(|00\rangle+|11\rangle)
$$

We consider each state separately, because the second requires purification after passing down a watched amplitude damping channel, but the first does not.

$$
\text { 1. The case } \frac{1}{\sqrt{2}}(|01\rangle+|10\rangle)
$$

Initially, consider sending a state $\frac{1}{\sqrt{2}}(|01\rangle+|10\rangle)$ down the channel with $N$ sources of entangled qubits at the precise midpoint of the channel as shown below:

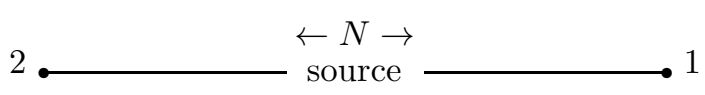

In the above diagram, let 1 and 2 symbolize two qubits from one of the sources reaching opposite ends of the channel. If both the qubits pass through an amplitude damping channel on their way to the ends of the channel, the final state of the qubits and their environment is:

$$
\begin{aligned}
|\Psi\rangle_{12 E_{1} E_{2}} & =\frac{1}{\sqrt{2}}\left(e^{-\Gamma}\left[|01\rangle_{12}+|10\rangle_{12}\right]|00\rangle_{E_{1} E_{2}}\right. \\
& \left.+\sqrt{1-e^{-2 \Gamma}}|00\rangle_{12}[|10\rangle+|01\rangle]_{E_{1} E_{2}}\right)
\end{aligned}
$$

If the environment is being watched then there is probability $e^{-2 \Gamma}$ that the resulting state is unchanged and maximally entangled, and $1-e^{-2 \Gamma}$ that it is a (useless) product state. In this way we will end up with

$$
K_{0}=N e^{-2 \Gamma}
$$

surviving entangled pairs, where the subscript 0 has been used to indicate that the channel has no repeaters (is undivided). Consider now splitting the channel into two and placing half of the sources at the midpoint of one half and the rest half of the sources in the midpoint of the other half in the following manner:

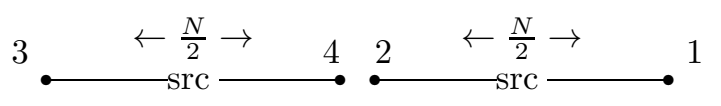

In the above diagram, let 1 and 2 stand for the qubits from any one of the sources reaching the ends of the right half-channel and 3 and 4 stand for similar qubits reaching the ends of the left half-channel. The place where 2 and 4 meet, namely the midpoint of the full-channel, is the site of a quantum repeater. If we kept the total number $N$ of sources of entangled pairs fixed, but still large, then $N / 2$ pairs are now used for each half-channel. For each half, we now have $\Gamma \rightarrow \Gamma / 2$. This means the number of maximally entangled pairs available finally in each half is the product of $N / 2$ multiplied by the probability $\frac{1}{2} e^{-2 \Gamma / 2}$ of getting a maximally entangled state $i . e . \frac{N}{2} \times e^{-2 \Gamma / 2}$. Let particle pairs $(1,2)$ and $(3,4)$ in the above diagram now be taken to symbolize any two of the several maximally entangled pairs obtained in the half-channels. If joint projections are now performed on particles 2 and 4 by an operator at the repeater, a maximally entangled pair is obtained across the full channel. The number of maximally entangled pairs obtained finally across the whole channel is thus equal to the number of maximally entangled pairs obtained in each half-channel prior to the joint projections on 2 and 4 at the repeater. It is therefore equal to

$$
K_{1}=\frac{N}{2} \times e^{-2 \Gamma / 2},
$$

where the subscript 1 denotes the fact that one repeater is now being used. So, whilst the exponent of $K_{1}$ is smaller compared to that of $K_{0}$, there is an extra factor of $1 / 2$ in front of $K_{1}$ compared to $K_{0}$. So is $K_{0}$ larger or smaller than $K_{1}$ ? Fig. 3 plots $K_{0}$ and $K_{1}$ as functions of $\Gamma$. This shows that after a certain value of $\Gamma$, it is better (in terms of obtaining higher final entanglement) to split the 
full-channel to two half-channels with a repeater in the middle.

This idea can be generalized to splitting the channel into $m$ sections. Eq.(2) can then be generalized to:

$$
K_{m-1}=\frac{N}{m} \times e^{-2 \Gamma / m}
$$

In each case, after a certain value of $\Gamma$, it becomes better to use 3 rather than 2 or 4 rather than 3 etc. sections of the channel in order to obtain maximum entanglement.

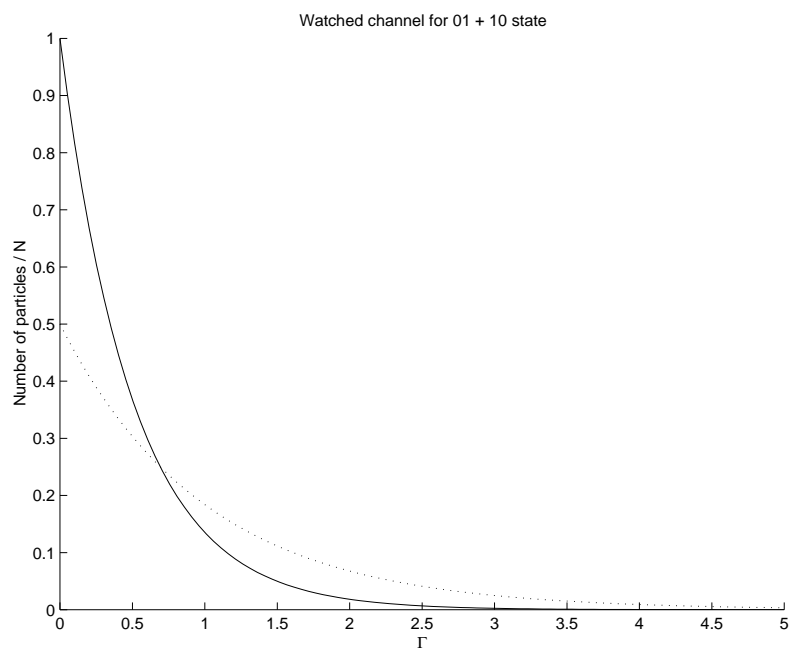

FIG. 3. The figure shows the variation of the number of maximally entangled pairs established between the ends of a watched amplitude damping channel following two different methods of distribution. The bold line shows the case of a channel without repeaters and the dotted line shows the case of a channel with one repeater. In both cases, the same number of initial sources of entanglement are used and all these sources emit the state $|01\rangle+|10\rangle$.

We now show how this idea can be used to achieve a resource reduction in entanglement distribution via repeaters (i.e., via splitting of a channel into several segments). We will show that we will need a smaller number of sources of entangled pairs to obtain the same amount of final entanglement across two ends of the channel. Consider relabeling $N$ in Eq.(3) to $N^{\prime}$ and setting that expression equal to $K_{0}$. This condition demands that with $m-1$ repeaters and $N^{\prime}$ sources of entanglement we produce the same number of maximally entangled pairs finally across the channel as the undivided channel with $N$ sources placed in the middle. We can then compare the ratio $N / N^{\prime}$, that is, the ratio of input pairs (sources) required for the undivided channel to the number of input pairs (sources) required for the channel with repeater stations. Let us label this ratio $\eta$. Then we have

$$
\eta=\frac{e^{-2 \Gamma / m}}{m \times e^{-2 \Gamma}}
$$

When $\eta$ is greater than one, it means the channel with repeaters needs fewer input pairs (sources) i.e. less re- sources. Graph 1 plots $\eta$ versus $m$ - the number of sections in a channel with repeaters, for a fixed value of $\Gamma=1.5$. At $m=3$ sections (or two repeaters), the resource reduction over the undivided channel is highest. Two repeaters is thus the the optimal way to distribute entanglement for $\Gamma=1.5$. In this case, using just $\frac{2}{5}$ th of the amount of initial resources, we achieve the same output of entanglement as an undivided channel.

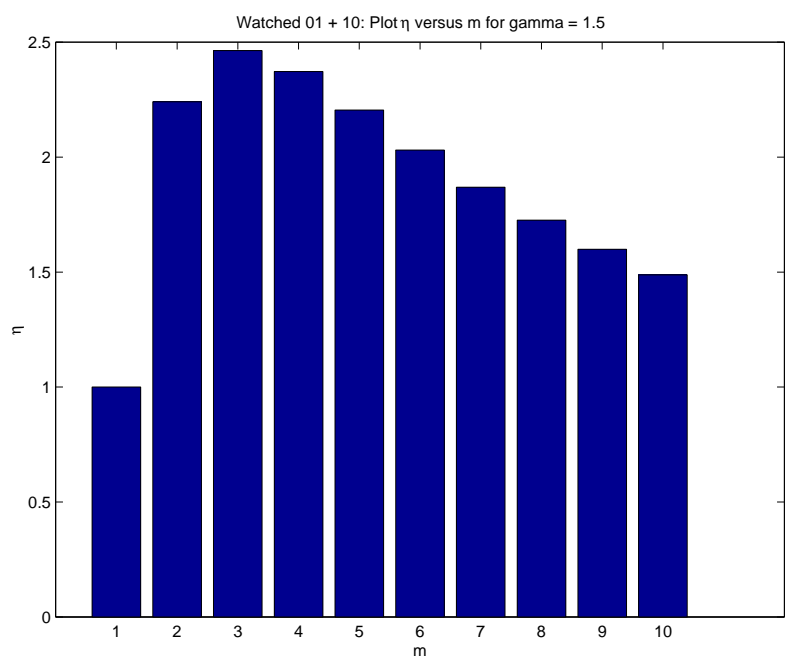

FIG. 4. The resource reduction ratio $\eta$ for a watched amplitude damping channel is shown for various values of the number $m$ of sections of the channel (when repeaters are used). The state emitted by the sources is taken to be $|01\rangle+|10\rangle$ and $\Gamma=1.5$.

Fig. 5 is a plot in 3D, extending Fig 4 over a range of $\Gamma$ to show the variation of the optimal number of divisions of the channel with $\Gamma$. We see that as $\Gamma$ (which is proportional to the channel length) increases, it becomes more and more advantageous in terms of resource reduction to use repeaters.

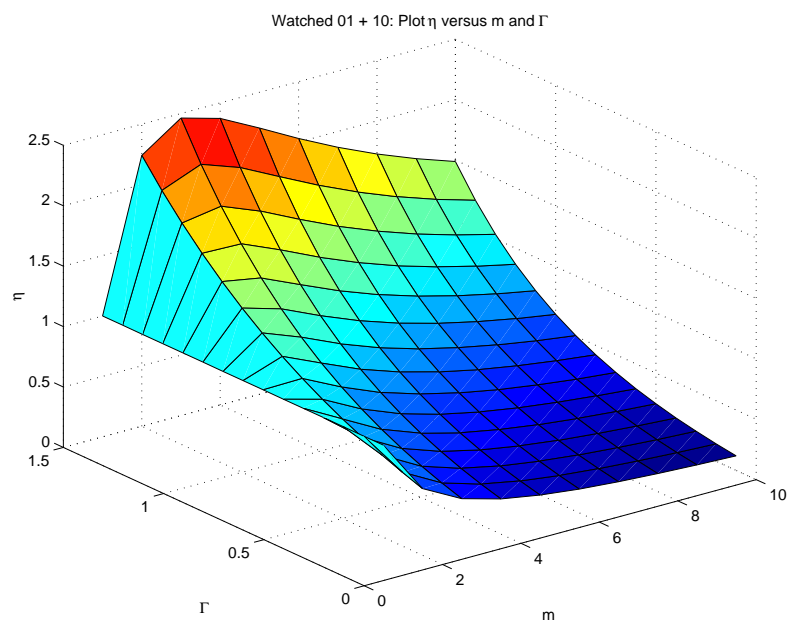


FIG. 5. The resource reduction ratio $\eta$ for a watched amplitude damping channel is shown for various values of the number $m$ of sections of the channel (when repeaters are used) and various values of $\Gamma$. The state emitted by the sources is taken to be $|01\rangle+|10\rangle$. The plot illustrates the fact that if the number of repeaters is kept fixed, more and more resource reduction is obtained with increase of the channel length.

\section{The case $\frac{1}{\sqrt{2}}(|00\rangle+|11\rangle)$}

We now consider sending two qubits in the initial state $\frac{1}{\sqrt{2}}(|00\rangle+|11\rangle)$ down an amplitude-damped channel. The resulting state is:

$$
\begin{aligned}
\left|\Psi^{\prime}\right\rangle_{12 E_{1} E_{2}} & =\frac{1}{\sqrt{2}}\left(\sqrt{1+e^{-4 \Gamma}}\left[\frac{|00\rangle+e^{-2 \Gamma}|11\rangle}{\sqrt{1+e^{-4 \Gamma}}}\right]_{12}|00\rangle_{E_{1} E_{2}}\right. \\
& +e^{-\Gamma} \sqrt{1-e^{-2 \Gamma}}|01\rangle_{12}|10\rangle_{E_{1} E_{2}} \\
& +e^{-\Gamma \sqrt{1-e^{-2 \Gamma}}|10\rangle_{12}|01\rangle_{E_{1} E_{2}}} \\
& \left.+\left(1-e^{2-\Gamma}\right)|00\rangle_{12}|11\rangle_{E_{1} E_{2}}\right)
\end{aligned}
$$

Then, if the environment is being monitored, there is a probability of $\frac{1}{2}\left(1+e^{-4 \Gamma}\right)$ that the state observed is (corresponds to the state $|00\rangle_{E_{1} E_{2}}$ of the environment)

$$
\left|\Psi_{c}^{\prime}\right\rangle_{12}=\frac{1}{\sqrt{1+e^{-4 \Gamma}}}\left(|00\rangle+e^{-2 \Gamma}|11\rangle\right)
$$

where the subscript $c$ represents the fact that this state is a result of conditional evolution. $\left|\Psi_{c}^{\prime}\right\rangle_{12}$ is not maximally entangled and must now be purified. When a large number of pure states like these are distilled, the number of maximally entangled states that can be produced is given by $N S\left(\rho^{r}\right)$ [16], where $\rho^{r}$ is the reduced density matrix of any one of the qubits and $S(\rho)$ is the von-Neumann entropy given by $-\operatorname{Tr} \rho \log _{2} \rho$.

If we began with an undivided channel and $N$ pairs were dispatched from $N$ sources, all placed at the midpoint, then $\frac{1}{2}\left(1+e^{-4 \Gamma}\right) N$ reach opposite ends of the channel in the state $\left|\Psi_{c}^{\prime}\right\rangle_{12}$ and rest reach the ends disentangled. One can locally purify these states to obtain

$$
K_{0}^{\prime}=\frac{1}{2}\left(1+e^{-4 \Gamma}\right) S\left(\rho^{r}\right) N
$$

maximally entangled pairs between the two ends of the channel.

Now consider splitting this channel into two halves as before. At the midpoint of each half we place $N / 2$ sources and make them emit maximally entangled pairs. The probability that an entangled state arrives at the ends of a half-channel is $\frac{1}{2}\left(1+e^{-4 \Gamma / 2}\right)$. Each half then purifies its pairs (independently of the other half). So the number of maximally entangled pairs in each half, after purification is

$$
K_{1}^{\prime}=\frac{1}{2}\left(1+e^{-4 \Gamma / 2}\right) S\left(\rho_{\frac{1}{2}}^{r}\right) \times \frac{N}{2}
$$

where $\rho_{\frac{1}{2}}^{r}$ is the reduced density matrix of one of the qubits in the state shared between any end and the repeater before purification. The subscript $1 / 2$ in $\rho_{\frac{1}{2}}^{r}$ has been used to indicate the fact that now each qubit has traveled half the distance it would have had to travel for an undivided channel. The disentangled pairs after purification are discarded and only the pairs in a maximally entangled state are kept. A projection by a central operator to connect up the ends of the full channel with one maximally entangled state now requires one pair from each half, so $K_{1}^{\prime}$ is also the number of maximally entangled pairs that can be produced between the two ends of the entire channel.

The number of maximally entangled states $K_{0}^{\prime}$ produced by an undivided channel and $K_{1}^{\prime}$ produced due to two half-channels is plotted in Fig.6. Analogous to the case of $|01\rangle+|10\rangle$, at a certain value of $\Gamma$, we get more final entanglement between the two ends of the channel if we split the channel and place repeaters. When these results are compared to distributing the state $|01\rangle+|10\rangle$ we see that they are not as good - i.e. using $|01\rangle+|10\rangle$ will give more maximally entangled states across the full channel.

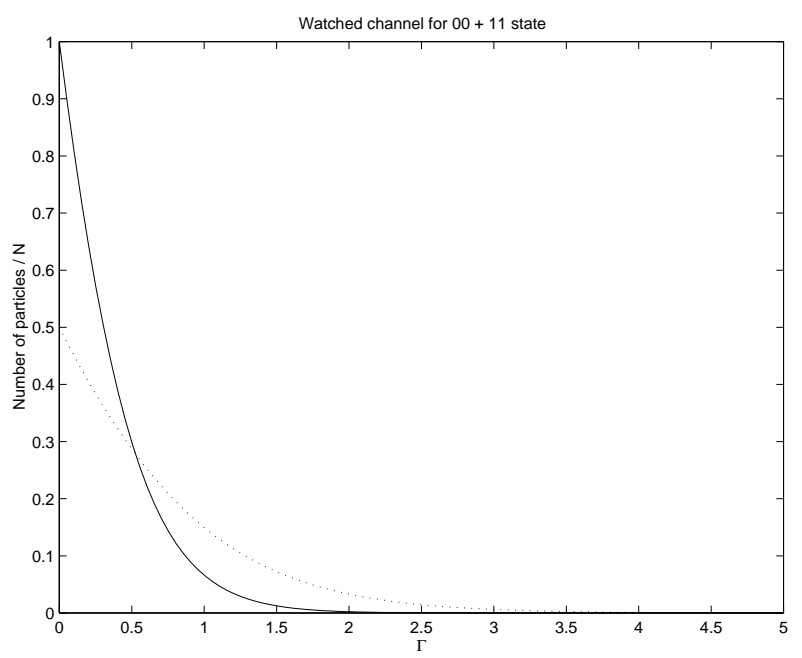

FIG. 6. The figure shows the variation of the number of maximally entangled pairs established between the ends of a watched amplitude damping channel following two different methods of distribution. The bold line shows the case of a channel without repeaters and the dotted line shows the case of a channel with one repeater. In both cases, the same number of initial sources of entanglement are used and all these sources emit the state $|00\rangle+|11\rangle$.

As before, this idea can be generalized to splitting the channel into $m$ sections. Each value of $m$ becomes the optimal number of channel sections (for obtaining the maximum final entanglement, if the initial resources were kept constant) after a certain value of $\Gamma$. Again, as before, we can calculate $\eta$, the ratio of the number of initial sources required for the undivided channel to the number of initial sources required for the channel with repeaters. 
Fig. 7 plots $\eta$ versus $m$ - the number of sections in the channel with repeaters, for $\Gamma=1$. We see that in this case, $m=3$ sections (two repeaters), gives the largest resource reduction over the undivided channel.

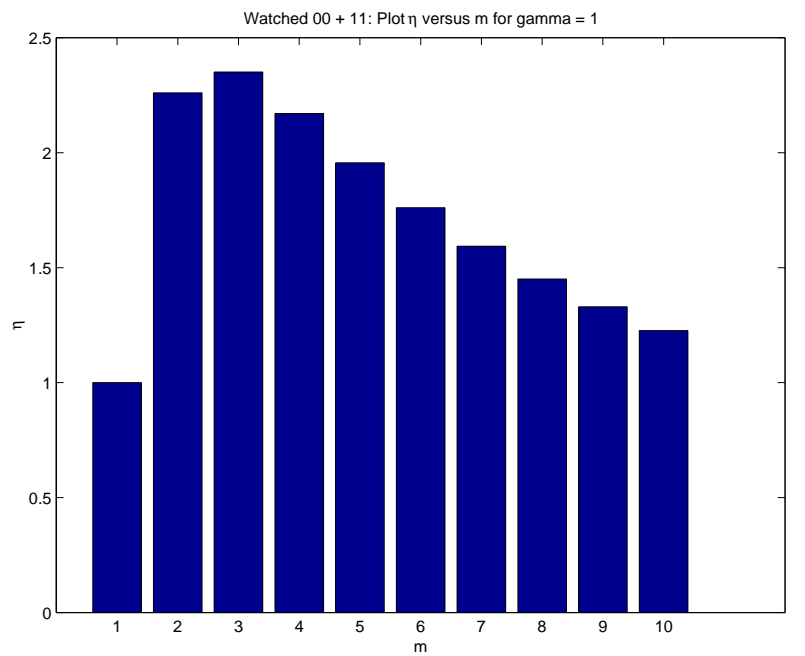

FIG. 7. The resource reduction ratio $\eta$ for a watched amplitude damping channel is shown for various values of the number $m$ of sections of the channel (when repeaters are used). The state emitted by the sources is taken to be $|00\rangle+|11\rangle$ and $\Gamma=1$.

\section{B. Bit-flip channel}

We now consider sending the states through a different kind of channel - a Pauli channel where there is just one type of error. The reason for this is the fact that the final states produced on sending a pair of qubits in a maximally entangled initial state through this channel is a mixture of two Bell states. The distillable entanglement for such states is exactly known [28,29]. If we take the single error in the channel to be of the "bitflip" type (called the bit-flip channel [2]), an initial state $\left|\psi^{+}\right\rangle=\frac{1}{\sqrt{2}}(|01\rangle+|10\rangle)$ transforms as

$$
\left|\psi^{+}\right\rangle\left\langle\psi^{+}|\rightarrow \lambda| \psi^{+}\right\rangle\left\langle\psi^{+}|+(1-\lambda)| \phi^{+}\right\rangle\left\langle\phi^{+}\right|,
$$

where $\left|\phi^{+}\right\rangle=\frac{1}{\sqrt{2}}(|00\rangle+|11\rangle)$. We relate the parameter $\lambda$ to the length of the channel (which is proportional to $\Gamma)$ by

$$
\lambda \equiv \frac{1+e^{-\Gamma}}{2} .
$$

From Ref. 28,29] we know that the distillable entanglement for the state $\Lambda=\lambda\left|\psi^{+}\right\rangle\left\langle\psi^{+}|+(1-\lambda)| \phi^{+}\right\rangle\left\langle\phi^{+}\right|$is given by $1-S(\Lambda)$, where $S(\Lambda)$ denotes the von Neumann entropy of the state $\Lambda$. On using an undivided channel with $N$ sources placed at the midpoint, we would obtain $\left.K_{0}^{\prime \prime}=N[1-S(\Lambda))\right]$ particle pairs in a final maximally entangled state across the full length of the channel. On the other hand, if we divided the channel into $m$ sections, and placed $N / m$ sources at the midpoint of each segment, the final number of maximally entangled pairs across the full channel will be $\left.K_{m}^{\prime \prime}=\frac{N}{m}\left[1-S\left(\Lambda_{m}\right)\right)\right]$ where $\Lambda_{m}=\lambda_{m}\left|\psi^{+}\right\rangle\left\langle\psi^{+}\left|+\left(1-\lambda_{m}\right)\right| \phi^{+}\right\rangle\left\langle\phi^{+}\right|$, in which $\lambda_{m}=\frac{1+e^{-\Gamma / m}}{2}$. In Fig. \&, we have plotted $K_{0}^{\prime \prime}$ and $K_{1}^{\prime \prime}$ as a function of $\Gamma$. It is clear that for $\Gamma$ exceeding a certain value, more final entanglement between the ends of the channel for a given initial resource is obtained on dividing the channel into two parts and using a repeater at their junction.

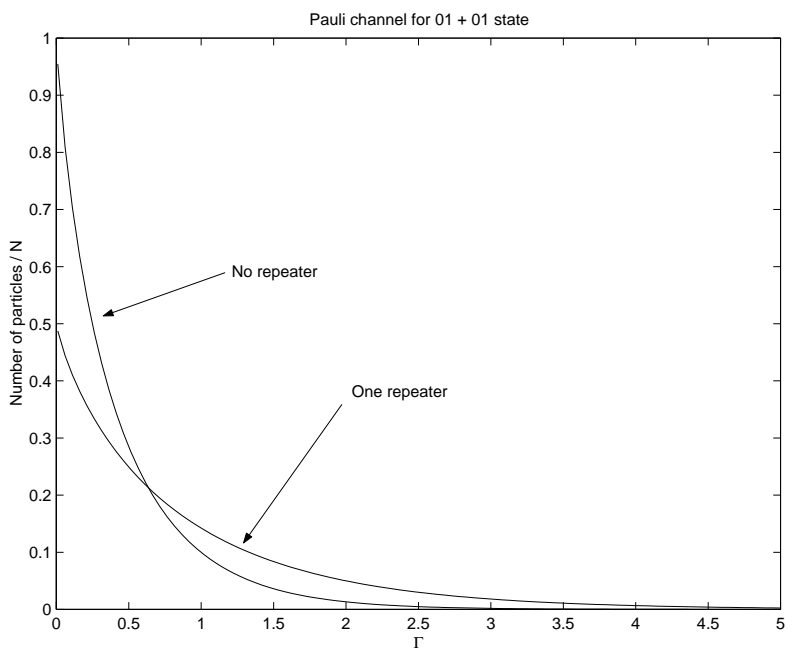

FIG. 8. The figure shows the variation of the number of maximally entangled pairs established between the ends of a bit-flip channel following two different methods of distribution: without repeaters and with one repeater. In both cases, the same number of initial sources of maximally entangled pairs are used.

As in the previous cases, we now consider how repeaters can be used to achieve the same final entanglement using less initial resources. The ratio of the number of initial sources required for the undivided channel to that required when the channel is split into $m$ sections with repeaters, is given by

$$
\eta=\frac{1-S\left(\Lambda_{m}\right)}{m(1-S(\Lambda))}
$$

This ratio $\eta$ is plotted in Fig (9) for $\Gamma=1.5$ and it can be seen that use of 1 repeater to 9 repeaters allow a smaller initial resource to create the same final amount of entanglement between the two ends of the full channel. 


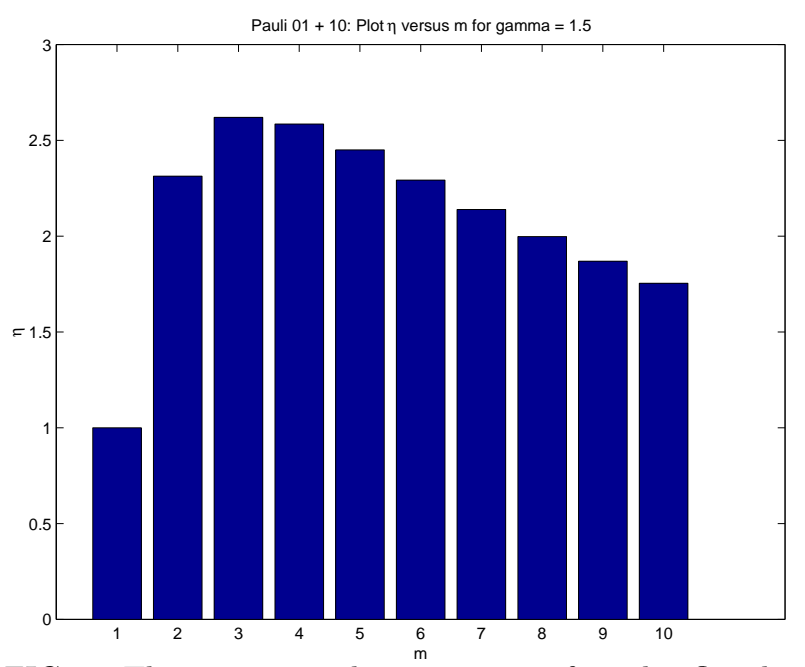

FIG. 9. The resource reduction ratio $\eta$ for a bit-flip channel is shown for various values of the number $m$ of sections of the channel (when repeaters are used). Here $\Gamma=1.5$.

Exactly the same results on resource reduction would hold for phase flip [3] and bit-phase flip [3] channels, as they all take initially maximally entangled states to final states which are mixtures of two Bell states.

\section{CONJECTURE BASED RESULTS}

We now consider more general channels. However, the final states produced when maximally entangled qubit pairs pass through these channels are such that their distillable entanglement is not known. For such channels we will use an established upper bound and a conjectured lower bound on distillable entanglement for comparing distribution with and without repeaters.

The well established upper bound on distillable entanglement $E_{D}$ is the entanglement of formation $E_{F}$ [17,26], which is also simple to calculate for a $2 \times 2$ system [26]. As a lower bound on distillable entanglement, we will use a conjecture recently made by Horodecki, Horodecki and Horodecki 30]. This gives a lower bound on the distillable entanglement of a state $\rho_{12}$ of qubits 1 and 2 by

$$
\left.E_{D} \geq \begin{array}{l}
S\left(\rho_{1}\right)-S\left(\rho_{12}\right) \\
S\left(\rho_{2}\right)-S\left(\rho_{12}\right)
\end{array}\right\} \text { whichever is lower, }
$$

where $\rho_{1}$ and $\rho_{2}$ denote the reduced density matrix of any one of the qubits. In Ref. [30], this conjecture has been used to investigate an unified approach to quantum channel capacities and there is a significant body of evidence which lends support to this conjecture [31].

For each type of channel, we first find the final density matrix $\rho(\Gamma)$ of two qubits for distribution through an undivided channel (with the source placed at the midpoint). We then calculate the density matrix $\rho(\Gamma / m)$ of entangled qubits reaching adjacent repeaters from the midpoints of the corresponding sections when the channel is split into $m$ sections. We now compare the lower bound $S\left(\rho_{1}\right)-S\left(\rho_{12}\right)$ or $S\left(\rho_{2}\right)-S\left(\rho_{12}\right)$ on distillable entanglement for the channel with repeaters with the upper bound $E_{F}$ for an undivided channel. In this way we ensure that we are imparting no intrinsic advantage to the case of repeaters over the case of an undivided channel. As before, the resource reduction is quantified by the ratio $\eta$ (defined in the same manner as earlier) and this ratio is given (assuming, without loss of generality, $S\left(\rho_{1}\right)$ to be the smaller among $S\left(\rho_{1}\right)$ and $\left.S\left(\rho_{2}\right)\right)$ by

$$
\eta=\frac{S\left(\rho_{1}(\Gamma / m)\right)-S\left(\rho_{12}(\Gamma / m)\right)}{m E_{F}(\rho(\Gamma))} .
$$

We consider three types of channels [2,3]

- Amplitude damping channel - i.e. the 'full' version of the watched channel

- Depolarising channel

- Phase damping channel

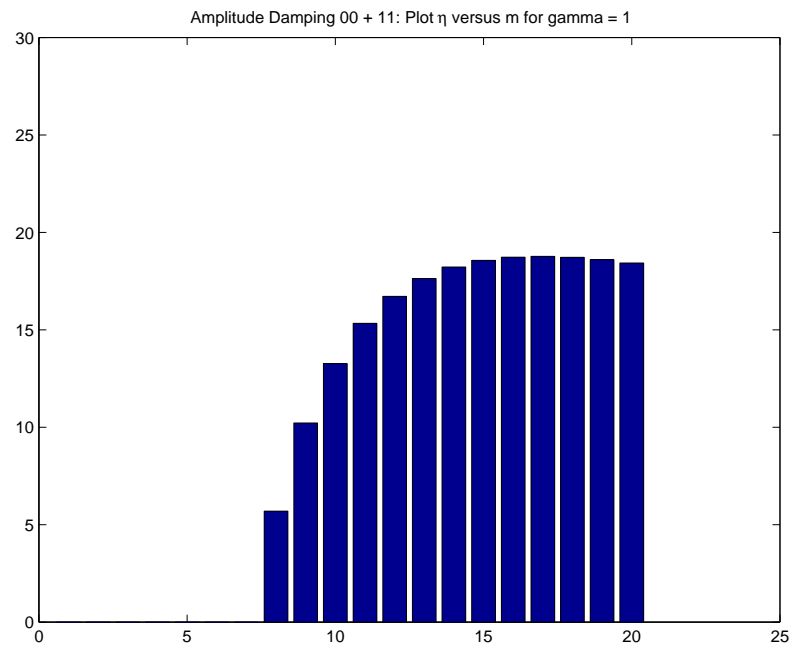

FIG. 10. The resource reduction ratio $\eta$ for an amplitude damping channel is shown for various values of the number $m$ of sections of the channel (when repeaters are used). The state emitted by the sources is taken to be $|00\rangle+|11\rangle$ and $\Gamma=1$. Before dividing the channel to 8 sections, no entanglement at all can be established between the ends of the channel. We see that $\eta$ increases at first with $m$ and then starts to decrease.

\section{A. Amplitude damping channel}

For the amplitude damping channel we consider sending two states down the channel, $\frac{1}{\sqrt{2}}(|01\rangle+|10\rangle)$ and $\frac{1}{\sqrt{2}}(|00\rangle+|11\rangle)$. This is because of the fact that the amplitude damping channel affects only the $|1\rangle$ state and therefore has quite different effects on the above two states. 
The amplitude damping channel affects states in the following way [2,3]

$$
\begin{aligned}
|0\rangle_{A}|0\rangle_{E} & \rightarrow|0\rangle_{A}|0\rangle_{E} \\
|1\rangle_{A}|0\rangle_{E} & \rightarrow \sqrt{1-p}|1\rangle_{A}|0\rangle_{E}+\sqrt{p}|0\rangle_{A}|1\rangle_{E} .
\end{aligned}
$$

We relate $p$ to $\Gamma$ by the following

$$
p=1-e^{-2 \Gamma}
$$

Fig.11. shows $\eta$ for $\frac{1}{\sqrt{2}}(|01\rangle+|10\rangle)$ and Fig. 10 for $\frac{1}{\sqrt{2}}(|00\rangle+$ $|11\rangle)$.

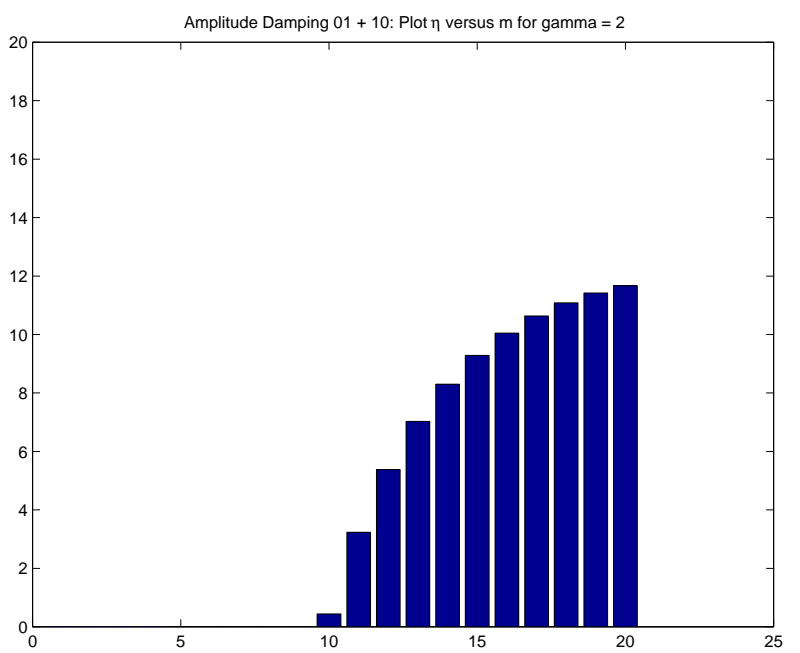

FIG. 11. The resource reduction ratio $\eta$ for an amplitude damping channel is shown for various values of the number $m$ of sections of the channel (when repeaters are used). The state emitted by the sources is taken to be $|01\rangle+|10\rangle$ and $\Gamma=2$. Before dividing the channel to 10 sections, no entanglement at all can be established between the ends of the channel and only from 11 sections onwards we see a resource reduction.

We see that in the case of using the state $\frac{1}{\sqrt{2}}(|01\rangle+$ $|10\rangle)$, there could almost be a 20 fold reduction of resources and in the case of the state $\frac{1}{\sqrt{2}}(|00\rangle+|11\rangle)$, there can be nearly a 12 fold reduction of resources if repeaters are used.

\section{B. Phase damping channel}

The phase damping channel affects states as [2] 3]

$$
\begin{aligned}
|0\rangle_{A}|0\rangle_{E} & \rightarrow \sqrt{1-p}|0\rangle_{A}|0\rangle_{E}+\sqrt{p}|0\rangle_{A}|1\rangle_{E} \\
|1\rangle_{A}|0\rangle_{E} & \rightarrow \sqrt{1-p}|1\rangle_{A}|0\rangle_{E}+\sqrt{p}|1\rangle_{A}|2\rangle_{E}
\end{aligned}
$$

We relate $p$ to $\Gamma$ by the following:

$$
p \rightarrow 1-e^{-\Gamma}
$$

This dependence of $p$ on $\Gamma$ has been so chosen that the entanglement of qubits propagating in this channel completely vanishes at $\Gamma \rightarrow \infty$. Fig. 12 shows $\eta$ for this channel.

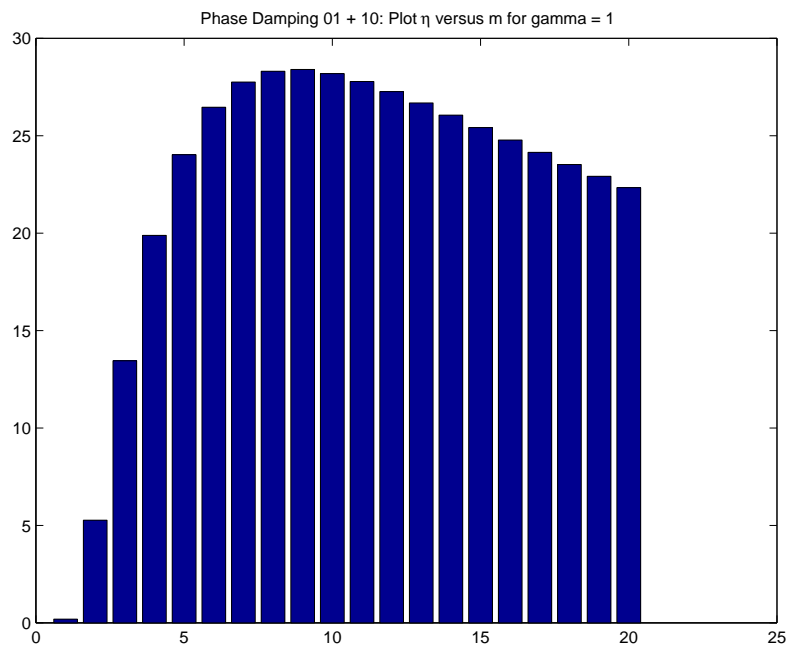

FIG. 12. The resource reduction ratio $\eta$ for a phase damping channel is shown for various values of the number $m$ of sections of the channel (when repeaters are used). Here $\Gamma=1$. As soon as the channel is split to 2 sections, there is a resource reduction and this first increases with $m$ and then starts decreasing again.

In this case we see that the use of repeaters can lead to almost up to 28 fold reduction in resources.

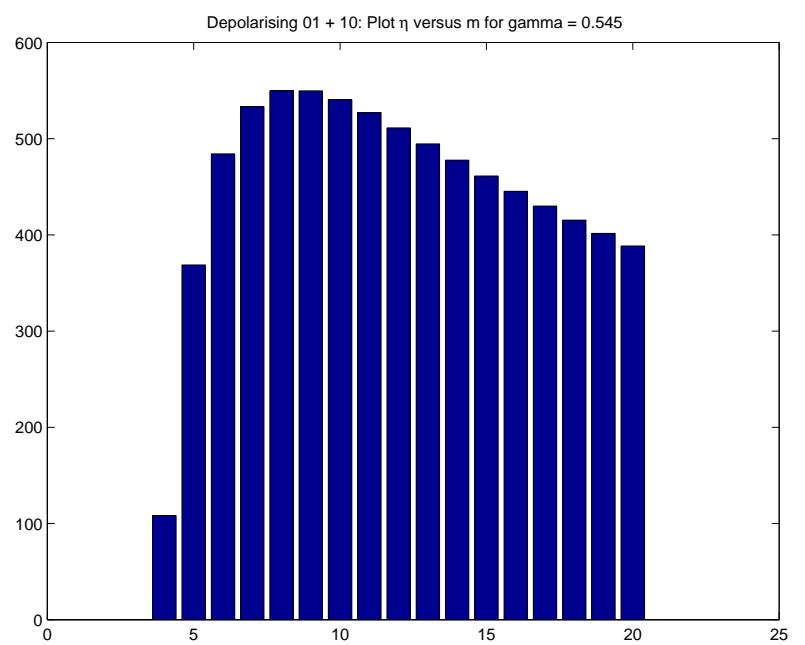

FIG. 13. The resource reduction ratio $\eta$ for a depolarizing channel is shown for various values of the number $m$ of sections of the channel (when repeaters are used). Here $\Gamma=0.545$. Before dividing up the channel to 4 sections, no entanglement can be established between its ends. The resource reduction ration $\eta$ initially increases with $m$ and then starts decreasing again. 


\section{Depolarizing channel}

The depolarizing channel affects states as

$$
\rho \rightarrow \rho^{\prime}=(1-p) \rho+\frac{p}{3}\left(\sigma_{1} \rho \sigma_{1}+\sigma_{2} \rho \sigma_{2}+\sigma_{3} \rho \sigma_{3}\right)
$$

We relate $p$ to $\Gamma$ by the following:

$$
p \rightarrow \frac{3\left(1-e^{-\Gamma}\right)}{4}
$$

This dependence of $p$ on $\Gamma$ has been so chosen that the entanglement of qubits propagating in this channel completely vanishes at $\Gamma \rightarrow \infty$. Fig. 13 shows $\eta$ for this channel.

In this case we see that use of repeaters can lead to, in the best case, a reduction as large as $500-600$ fold in resources. In the case of all channels, we see that the degree of resource reduction increases at first with the number of repeater stations and then starts to decrease on further increase of the number of repeaters.

\section{CONCLUSIONS}

We have shown that the amount of initial resource of entanglement needed to establish a certain final amount of entanglement between two ends of a noisy channel can be reduced, for certain lengths of the channel, by using quantum repeaters. This result has important bearing on cost minimization in entanglement distribution. We have used a variety of channels and asymptotic values and bounds on distillable entanglement to arrive at our results. While in the original papers on quantum repeaters 24], the emphasis was on preventing the exponential degradation of entanglement through practically motivated specific purification procedures, our emphasis is more on attempting to use general results on distillable entanglement. As such, our results are quite independent of the type of the purification procedures used. We also use asymptotic measures of entanglement [17,26 28] in our analysis in contrast to the earlier treatment 24] based on fidelity of transmission of the states. Though the field of entanglement measures is very well developed, there have not been many attempts (apart from the natural application to entanglement purification [28]) to link these measures to practical issues such as the cost of entanglement distribution. We regard our analysis to be a step in this direction. All our analysis has been based on asymptotic purification procedures in order to use simple entropic quantities as bounds on distillable entanglement. However, the field of local entanglement manipulations for non-asymptotic cases has developed recently [32. It would be interesting to explore the possibility of resource reduction by repeaters in the case when only a small number of entangled qubit pairs are transmitted through the channel at a time.
AH thanks the UK EPSRC (Engineering and Physical Sciences Research Council) for financial support.

[1] C. H. Bennett and D. P. DiVincenzo, Nature 404, 247 (2000); M.B. Plenio and V. Vedral, Cont. Phys. 39, 431 (1998); V. Vedral, The role of relative entropy in quantum information theory, quant-ph/0102094; A. Zeilinger, Phys. World 11, 35 (1998).

[2] M. A. Nielsen and I. L. Chuang, Quantum Computation and Quantum Information, Cambridge University Press, 2000.

[3] J. Preskill, Physics 229: Advanced Mathematical Methods of Physics - Quantum Computation and Information, California Institute of Technology, 1998. URL:http://www.theory.caltech.edu/people/preskill/ph229,.

[4] A. K. Ekert, Phys. Rev. Lett. 67, 661 (1991).

[5] C. H. Bennett, G. Brassard, C. Crepeau, R. Jozsa, A. Peres and W. K. Wooters, Phys. Rev. Lett. 70, 1895 (1993).

[6] C. H. Bennett and S. J. Wiesner, Phys. Rev. Lett. 69, 2881 (1992).

[7] K. Mattle, H. Weinfurter, P. G. Kwiat and A. Zeilinger, Phys. Rev. Lett. 76, 4656 (1996); D. Bouwmeester, J-W. Pan, K. Mattle, M. Eibl, H. Weinfurter, and A. Zeilinger, Nature (London) 390, 575 (1997); D. Boschi, S. Branca, F. De Martini, L. Hardy and S. Popescu, Phys. Rev. Lett. 80, 1121 (1998); A. Furasawa, J.L.Sørensen, S.L. Braunstein, C.A.Fuchs, H.J.Kimble and E.S.Polzik, Science 282, 706 (1998); W. Tittel, J. Brendel, H. Zbinden and N. Gisin, Phys. Rev. Lett. 84, 4737 (2000); T. Jennewein, C. Simon, G. Weihs, H. Weinfurter and A. Zeilinger, Phys. Rev. Lett. 84, 4729 (2000).

[8] C. H. Bennett and G. Brassard, International Conference on Computers, Systems and Signal Processing, Bagalore, India, December 10-12, 1984, pp 175-179.

[9] R. Cleve and H. Buhrman, Phys. Rev. A 56, 1201 (1997).

[10] A. Karlsson, M. Koashi and N. Imoto, Phys. Rev. A 59, 162 (1999); M. Hillery, V. Buzek and A. Berthiaume, Phys. Rev. A 59, 1829 (1999); R. Cleve, D. Gottesman and H. K. Lo, Phys. Rev. Lett. 83, 648 (1999); A. Cabello, Multiparty key distribution and secret sharing based on entanglement swapping, quant-ph/0009025.

[11] D. Deutsch, Proc. R. Soc. Lond. A 400, 97 (1985); P. Shor, SIAM Journal on Computing, 26, 1484 (1997). Also FOCS'94; L. Grover. A fast quantum mechanical algorithm for database search, Proceedings of the 28th ACM Symposium on Theory of Computing, p. 212, 1996. Also quant-ph/9605043.

[12] J. I. Cirac, A. K. Ekert, S. F. Huelga and C. Macchiavello, Phys. Rev. A 59, 4249 (1999).

[13] L. K. Grover, Quantum Telecomputation, quant$\mathrm{ph} / 9704012$.

[14] M. Murao, D. Jonathan, M.B. Plenio, and V. Vedral, Phys. Rev. A 59, 156 (1999); W. Dür and J. I. Cirac, J. Mod. Opt. 47, 247 (2000); M. Murao, M. B. Plenio 
and V. Vedral, Phys. Rev. A 61, 032311 (2000); A. K. Pati, Quantum cobweb: remote shared-entangling of an unknown quantum state, quant-ph/0101049.

[15] R. Jozsa, D. S. Abrams, J. P. Dowling, and C. P. Williams, Phys. Rev. Lett. 85, 2010 (2000); I. L. Chuang, Phys. Rev. Lett. 85, 2006 (2000); S. Bose, S. F. Huelga and M. B. Plenio, Phys. Rev. A 63, 032313 (2001).

[16] C. H. Bennett, H. J. Bernstein, S. Popescu and B. Schumacher, Phys. Rev. A 53, 2046 (1996); N. Gisin, Phys. Lett. A 210, 151 (1996).

[17] C. H. Bennett, D. P. DiVincenzo, J. A. Smolin and W. K. Wootters, Phys. Rev. A 54, 3824 (1996).

[18] C. H. Bennett, G. Brassard, S. Popescu, B. Schumacher, J. A. Smolin and W. K. Wootters, Phys. Rev. Lett. 76, 722 (1996); D. Deutsch, A. Ekert, R. Jozsa, C. Macchiavello, S. Popescu and A. Sanpera, Phys. Rev. Lett. 77, 2818 (1996).

[19] M. Murao, M. B. Plenio, S. Popescu, V. Vedral and P. L. Knight, Phys. Rev. A 57, R4075 (1998); W. Dür, J. I. Cirac and R. Tarrach, Phys. Rev. Lett. 83, 3562 (1999).

[20] E. Biham, B. Huttner and T. Mor, Phys. Rev. A 54, 2651 (1996).

[21] M. Zukowski, A. Zeilinger, M. Horne and A. K. Ekert, Phys. Rev. Lett. 71, 4287 (1993).

[22] S. Bose, V. Vedral and P. L. Knight, Phys. Rev. A 57, 822 (1998).

[23] S. Bose, V. Vedral and P. L. Knight, Phys. Rev. A 60, 194 (1999); L. Hardy and D. D. Song, Phys. Rev. A 62, 052315 (2000); B.-S. Shi, Y.-K. Jiang and G.-C. Guo, Phys. Rev. A 62, 054301 (2000); M. Cinchetti and J. Twamley, Entanglement distribution between $N$ distant users via a center (To appear in Phys. Rev. A).

[24] H.-J. Briegel, W. Dür, J. I. Cirac and P. Zoller 815932 (1998); W. Dür, H.-J. Briegel, J. I. Cirac and P. Zoller,
Phys. Rev. A 59, 169 (1999).

[25] J.-W. Pan, D. Bouwmeester, H. Weinfurter, and A. Zeilinger, Phys. Rev. Lett. 80, 3891 (1998); P. G. Kwiat, S. Barraza-Lopez, A. Stefanov and N. Gisin, Nature 409, 1014 (2001); J.-W. Pan, C. Simon, C. Brukner and A. Zeilinger, Feasible Entanglement Purification for Quantum Communication, quant-ph/0012026.

[26] S. Hill and W. K. Wootters, Phys. Rev. Lett. 78, 5022 (1997); W. K. Wootters, Phys. Rev. Lett. 80, 2245 (1998).

[27] V. Vedral, M. B. Plenio, M. A. Rippin and P. L. Knight, Phys. Rev. Lett. 78, 2275 (1997).

[28] V. Vedral and M. B. Plenio, Phys. Rev. A 57, 1619 (1998).

[29] E. M. Rains Phys. Rev. A 60, 179 (1999).

[30] M. Horodecki, P. Horodecki and R. Horodecki, Phys. Rev. Lett. 85, 433 (2000).

[31] M. B. Plenio, S. Virmani, and P. Papadopoulos, J. Phys. A 33, L193-197 (2000); J. Eisert, T. Felbinger, P. Papadopoulos, M. B. Plenio and M. Wilkens, Phys. Rev. Lett. 84, 1611 (2000); S. Bose, M. B. Plenio, and V. Vedral, J. Mod. Opt. 47, 291 (2000); G. Bowen, Phys. Rev. A 63, 022302 (2001); T. Hiroshima, Optimal dense coding with mixed state entanglement, quant-ph/0009048.

[32] H.-K. Lo and S. Popescu, Phys. Rev. A 63, 022301 (2001); M. A. Nielsen, Phys. Rev. Lett. 83, 436 (1999); G. Vidal, Phys. Rev. Lett. 83, 1046 (1999); D. Jonathan and M. B. Plenio, Phys. Rev. Lett. 83, 1455 (1999); L. Hardy, Phys. Rev. A 60, 1912 (1999); D. Jonathan and M. B. Plenio, Phys. Rev. Lett. 83, 3566 (1999); G. Vidal, D. Jonathan and M. A. Nielsen, Phys. Rev. A 62, 012304 (2000); J. Eisert and M. Wilkens, Phys. Rev. Lett. 85, 437 (2000); A. Acin, E. Jane, W. Dür and G. Vidal, Phys. Rev. Lett. 85, 4811 (2000). 